\title{
CONTINUOUSLY PARAMETRIZED BESICOVITCH SETS IN $\mathbf{R}^{n}$
}

\author{
Esa Järvenpää, Maarit Järvenpää, Tamás Keleti and András Máthé \\ University of Oulu, Department of Mathematical Sciences \\ P.O. Box 3000, 90014 University of Oulu, Finland; Esa.Jarvenpaa@oulu.fi \\ University of Oulu, Department of Mathematical Sciences \\ P.O. Box 3000, 90014 University of Oulu, Finland; Maarit.Jarvenpaa@oulu.fi \\ Eötvös Loránd University, Department of Analysis \\ Pázmány Péter sétány 1/C, H-1117 Budapest, Hungary; elek@cs.elte.hu \\ University of Warwick, Department of Mathematics \\ Coventry CV4 7AL, United Kingdom; A.Mathe@warwick.ac.uk
}

\begin{abstract}
We study continuous 1-dimensional time parametrization and $(n-1)$-dimensional direction parametrization of Besicovitch sets in $\mathbf{R}^{n}$. In the 1-dimensional case we prove that for $n \geq 3$ one can move a unit line segment (in fact even a full line) continuously in $\mathbf{R}^{n}$ within a set of measure zero in such a manner that the line segment points in all possible directions. We also show that in $\mathbf{R}^{n}$, for any $n \geq 2$, one can parametrize unit line segments continuously by their direction so that all segments are contained in a set of arbitrarily small measure. However, if we parametrize lines continuously by their direction then the set which is not covered by their union is bounded.
\end{abstract}

\section{Introduction}

In 1917 Kakeya [8] asked the following question.

Kakeya needle problem. What is the least amount of area required to rotate continuously a unit line segment in the plane by a full rotation (i.e. by $360^{\circ}$ )?

Not knowing about this question, Besicovitch [1] constructed almost at the same time a planar set of Lebesgue measure zero that contains a unit segment in every direction. A set containing a unit segment in every direction is called a Besicovitch set. Later [2] he verified that a modification of his construction gives the following answer to the Kakeya needle problem: there exists a planar set of arbitrarily small area within which a unit line segment can be continuously rotated by a full rotation.

Is it possible to rotate a unit line segment continuously within a planar set of measure zero? It was already mentioned by Besicovitch in a footnote of [2] that this is impossible but a proof was published only recently by Tao [11]. Then O'Neil [10] proved that there exists a planar set of Lebesgue measure zero within which a line segment can be rotated by a map that is of Baire class 1, that is, a pointwise limit of a sequence of continuous functions.

doi:10.5186/aasfm.2011.3639

2010 Mathematics Subject Classification: Primary 28A75, 51M15, 51M25.

Key words: Besicovitch set, Kakeya needle problem, continuous parametrization, measure, lines in every direction.

We acknowledge the support of the Centre of Excellence in Analysis and Dynamics Research funded by the Academy of Finland. The fourth author was supported by Hungarian Scientific Foundation grant no. 72655 . 
In this paper we study the above questions in higher dimension. In Section 1 we show that in $\mathbf{R}^{n}$ for $n \geq 3$ one can do more than in the plane: it is possible to move a unit line segment (in fact even a full line) continuously within a set of measure zero in such a way that the line segment points in all possible directions. Hence, if we consider the time as the parameter of the continuous rotation then the above result gives a positive answer to the question. This answer leads to the natural question whether one can construct a continuous map from the set of directions to the set of unit line segments of $\mathbf{R}^{n}$ (or to the set of lines in $\mathbf{R}^{n}$ ) so that one assigns to each direction a unit line segment (or line) in that direction and all line segments (or lines) are contained in a small set. In Section 2 we verify that this is not possible for lines: the union of the lines is the whole $\mathbf{R}^{n}$ if $n$ is odd and it has bounded complement if $n$ is even. However, as we show in Section 3, for unit line segments such a continuous parametrization by the direction is possible inside a set of arbitrarily small measure.

The main tools we use are the Besicovitch projection theorem in Section 1, algebraic topology in Section 2 and a simply connected construction given by Cunningham [4] to the Kakeya needle problem in Section 3.

Finally, we remark that this work (as the question and the work of Kakeya as well) has almost nothing to do with the famous "Kakeya conjecture", which states that Besicovitch sets in $\mathbf{R}^{n}$ must have Hausdorff dimension $n$.

\section{Time parametrization}

We start by giving an explicit construction of a continuously parametrized Besicovitch set in $\mathbf{R}^{n}$ for any $n \geq 3$.

Theorem 1.1. For any $n \geq 3$ one can continuously move a line in a closed subset of $\mathbf{R}^{n}$ having zero Lebesgue measure so that the line points in every direction. More precisely, there exists a continuous map $L:[0,1] \rightarrow\left\{\right.$ lines of $\left.\mathbf{R}^{n}\right\}$ such that

(i) for every line $l$ of $\mathbf{R}^{n}$ there exists a $t \in[0,1]$ so that $L(t)$ is parallel to $l$, and

(ii) the set $\bigcup_{t \in[0,1]} L(t)$ is a closed set of Lebesgue measure zero.

Proof. For any given $u, w \in\{1\} \times[0,1]^{n-1}$ we construct a map $L$ that has all the required properties but instead of (i) the following holds:

(i') The lines $L(0)$ and $L(1)$ are parallel to $u$ and $w$, respectively, and for any $v \in\{1\} \times[0,1]^{n-1}$ there exists $t \in[0,1]$ so that $L(t)$ is parallel to $v$.

Since every line through the origin hits at least one of the faces of $[-1,1]^{n}$, say $V$, and therefore at least one translate of the $(n-1)$-dimensional unit cube inside $V$ having a vertex at the center of $V$, by gluing finitely many such maps and by rescaling we will get the required map.

The following set will code the lines we take. Let

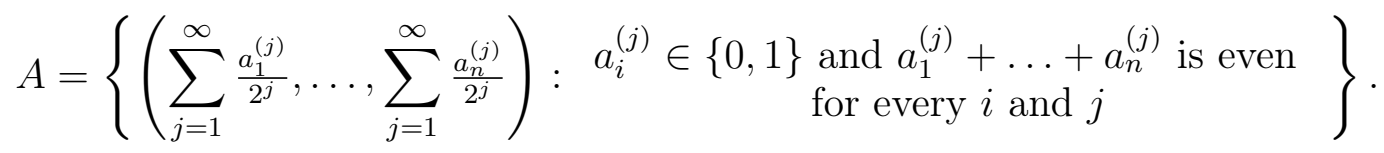

Define $A_{0}=[0,1]^{n}$ and

$$
A_{k}=\left\{\left(\sum_{j=1}^{\infty} \frac{a_{1}^{(j)}}{2^{j}}, \ldots, \sum_{j=1}^{\infty} \frac{a_{n}^{(j)}}{2^{j}}\right): \begin{array}{l}
a_{i}^{(j)} \in\{0,1\} \text { for every } i \text { and } j \text { and } \\
a_{1}^{(j)}+\ldots+a_{n}^{(j)} \text { is even for } j=1, \ldots, k
\end{array}\right\}
$$


for all $k=1,2, \ldots$ Note that $A=\bigcap_{k=0}^{\infty} A_{k}$ and $A_{k}$ is the union of $2^{(n-1) k}$ closed cubes of side length $2^{-k}$ with disjoint interiors that are obtained from the cubes of $A_{k-1}$ by considering a black and white $2 \times \ldots \times 2$ chessboard on each cube of $A_{k-1}$ so that the little cube closest to the origin is always black and by taking the black ones. Both constructions show that $A$ is a self-similar set. One of the important properties of $A$ is the following:

Claim 1.2. For any $v=\left(1, a_{2}, \ldots, a_{n}\right) \in\{1\} \times[0,1]^{n-1}$ there exists $a_{1} \in[0,1]$ so that $\left(a_{1}, \ldots, a_{n}\right) \in A$.

Proof. The claim follows easily from the definition of $A$ : Write $a_{2}, \ldots, a_{n}$ in binary form as $a_{i}=\sum_{j=1}^{\infty} a_{i}^{(j)} 2^{-j}(i=2, \ldots, n)$ and for each $j$ choose $a_{1}^{(j)} \in\{0,1\}$ so that $a_{1}^{(j)}+\ldots+a_{n}^{(j)}$ is even. Then for $a_{1}=\sum_{j=1}^{\infty} a_{1}^{(j)} 2^{-j}$ we clearly have $\left(a_{1}, \ldots, a_{n}\right) \in$ A.

By this claim, we can choose $u^{\prime}, w^{\prime} \in A$ for the given $u, w \in\{1\} \times[0,1]^{n-1}$ so that changing the first coordinates of $u^{\prime}$ and $w^{\prime}$ to 1 (if necessary) we get $u$ and $w$, respectively.

Now we construct a Peano type curve with range $A$ that starts from $u^{\prime}$ and ends at $w^{\prime}$.

Claim 1.3. There exists a continuous and onto map $\Gamma:[0,1] \rightarrow A$ so that $\Gamma(0)=$ $u^{\prime}$ and $\Gamma(1)=w^{\prime}$.

Proof. The construction is similar to the classical Peano curve construction. One can easily check that the intersection of any two cubes of $A_{1}$ contains at least one point of $A$, and so the same holds for the little cubes of $A_{k}$ that are contained in a fixed cube of $A_{k-1}$.

Let $\Gamma(0)=u^{\prime}$ and $\Gamma(1)=w^{\prime}$. We enumerate the $2^{n-1}$ cubes of $A_{1}$ so that the first one contains $u^{\prime}$ and the last one contains $w^{\prime}$. We allow repetition if necessary: the first one and the last one may be the same. Then we divide $[0,1]$ into $2^{n-1}$ or $2^{n-1}+1$ intervals of equal length. We match the $m^{\text {th }}$ interval to the $m^{\text {th }}$ cube of $A_{1}$ and define $\Gamma(t)$ at the touching points of the intervals as a point of the intersection of $A$ and the corresponding two cubes.

We continue by induction. At the $k^{\text {th }}$ step, in each cube of $A_{k-1}$ we enumerate the little cubes of $A_{k}$ again allowing repetition so that the first one contains the already chosen image of the left endpoint of the corresponding interval defined at the previous step and the last one contains the image of the right endpoint. Then we subdivide each interval of the previous step into $2^{n-1}$ or $2^{n-1}+1$ intervals of equal length, match them to the enumerated little cubes of the corresponding cube of $A_{k}$ in the given order and define $\Gamma(t)$ at the touching points of the new intervals as a point of the intersection of $A$ and the corresponding two cubes. Finally, we extend $\Gamma$ continuously to $[0,1]$.

Now we can define the map $L$ as follows. Let

$$
L(t)=l(\Gamma(t)), \text { where } l\left(\left(a_{1}, \ldots, a_{n}\right)\right)=\left\{\left(h, a_{1}+a_{2} h, a_{3} h, \ldots, a_{n} h\right): h \in \mathbf{R}\right\} .
$$

Then $L$ is clearly a continuous map from $[0,1]$ to $\left\{\right.$ lines of $\left.\mathbf{R}^{n}\right\}$.

First we prove (i'). By construction, if $\Gamma(t)=\left(a_{1}, \ldots, a_{n}\right)$ then $L(t)$ is parallel to $\left(1, a_{2}, \ldots, a_{n}\right)$. Since $L(0)=l(\Gamma(0))=l\left(u^{\prime}\right)$ and $L(1)=l\left(w^{\prime}\right)$, this clearly implies 
that the lines $L(0)$ and $L(1)$ are parallel to $u$ and $w$, respectively. The other part of (i') follows from Claim 1.2.

Now we prove (ii). Let $F=\bigcup_{t \in[0,1]} L(t)$. By definition,

$$
F=\left\{\left(h, a_{1}+a_{2} h, a_{3} h, \ldots, a_{n} h\right):\left(a_{1}, \ldots, a_{n}\right) \in A, h \in \mathbf{R}\right\},
$$

which is a closed subset of $\mathbf{R}^{n}$ since $A$ is compact. Define

$$
A_{a_{3}, \ldots, a_{n}}=\left\{\left(a_{1}, a_{2}\right):\left(a_{1}, \ldots, a_{n}\right) \in A\right\} .
$$

Claim 1.4. For almost every $\left(a_{3}, \ldots, a_{n}\right) \in[0,1]^{n-2}$ the set $A_{a_{3}, \ldots, a_{n}}$ is purely unrectifiable and has finite length.

Proof. For almost every $\left(a_{3}, \ldots, a_{n}\right) \in[0,1]^{n-2}$ the set $A_{a_{3}, \ldots, a_{n}}$ has finite length since if $a_{3}, \ldots, a_{n}$ are irrational then for any $k$ it can be covered by $2^{k}$ squares of side length $2^{-k}$.

Assume that $a_{3}, \ldots, a_{n}$ can be written as $a_{i}=\sum_{j=1}^{\infty} a_{i}^{(j)} 2^{-j}(i=3, \ldots, n)$, where $a_{i}^{(j)} \in\{0,1\}$ for every $i$ and $j$, and $a_{3}^{(j)}+\ldots+a_{n}^{(j)}$ is even for infinitely many $j$ and odd for infinitely many $j$. Since almost every $\left(a_{3}, \ldots, a_{n}\right) \in[0,1]^{n-2}$ satisfies this condition it is enough to prove that $A_{a_{3}, \ldots, a_{n}}$ is purely unrectifiable under this assumption.

It is a well-known and fairly easy fact (see e.g. $[9,18.10(4)]$ ) that if a Borel set in the plane has two projections of zero Lebesgue measure then it is purely unrectifiable. Hence it is sufficient to show that $A_{+}=\left\{a_{1}+a_{2}:\left(a_{1}, a_{2}\right) \in A_{a_{3}, \ldots, a_{n}}\right\}$ and $A_{-}=\left\{a_{1}-a_{2}:\left(a_{1}, a_{2}\right) \in A_{a_{3}, \ldots, a_{n}}\right\}$ have measure zero.

Let $e_{1}, e_{2}, \ldots$ be those indices $j$ for which $a_{3}^{(j)}+\ldots+a_{n}^{(j)}$ is even and let $o_{1}, o_{2}, \ldots$ be those indices $j$ for which $a_{3}^{(j)}+\ldots+a_{n}^{(j)}$ is odd. Let $\left(a_{1}, a_{2}\right) \in A_{a_{3}, \ldots, a_{n}}$. Then, by the definition of $A, a_{1}$ and $a_{2}$ can be written as $a_{1}=\sum_{j=1}^{\infty} a_{1}^{(j)} 2^{-j}$ and $a_{2}=$ $\sum_{j=1}^{\infty} a_{2}^{(j)} 2^{-j}\left(a_{i}^{(j)} \in\{0,1\}\right)$, where $a_{1}^{\left(e_{k}\right)}=a_{2}^{\left(e_{k}\right)}$ and $a_{1}^{\left(o_{k}\right)}+a_{2}^{\left(o_{k}\right)}=1$ for every $k=1,2, \ldots$ Thus for any $\left(a_{1}, a_{2}\right) \in A_{a_{3}, \ldots, a_{n}}$ the number $\left(a_{1}+a_{2}-\sum_{k=1}^{\infty} 2^{-o_{k}}\right) / 2$ can be written in base 2 so that the digits labelled by $o_{1}, o_{2}, \ldots$ are all zeros and the number $\left(a_{1}-a_{2}+\sum_{k=1}^{\infty} 2^{-o_{k}}\right) / 2$ so that the digits labelled by $e_{1}, e_{2}, \ldots$ are all zeros. This implies that $A_{+}$and $A_{-}$are of measure zero, which completes the proof of the claim.

By the Besicovitch projection theorem [3] (see also [9, Theorem 18.1]) almost every projection of a purely unrectifiable Borel subset of the plane with finite length has zero Lebesgue measure. Thus, by Claim 1.4, for almost every $\left(a_{3}, \ldots, a_{n}\right) \in$ $[0,1]^{n-2}$ the set

$$
\left\{a_{1}+a_{2} h:\left(a_{1}, \ldots, a_{n}\right) \in A\right\}
$$

has zero measure for almost every $h \in \mathbf{R}$. Using Fubini's theorem, we see that $F$ has measure zero, which completes the proof of (ii) and also the proof of Theorem 1.1.

Corollary 1.5. For any $n \geq 3$ and $\varepsilon>0$ one can continuously move a unit line segment in $\mathbf{R}^{n}$ so that the line segment points in all possible directions within a compact subset of zero Lebesgue measure inside a ball of radius $\frac{1}{2}+\varepsilon$.

Proof. Let $L:[0,1] \rightarrow\left\{\right.$ lines of $\left.\mathbf{R}^{n}\right\}$ be a map provided by Theorem 1.1. First we take a continuous point-selection $P:[0,1] \rightarrow \mathbf{R}^{n}$ of $L$ by which we mean that $P$ is continuous and $P(t) \in L(t)$ for every $t \in[0,1]$. This can be done, for example, by taking for each $t$ the closest point of $L(t)$ to the origin. Since $P$ is continuous 
$P([0,1])$ is compact, and therefore, it is contained in a ball centered at the origin with some radius $r$. By shrinking everything from the origin by ratio $\frac{\varepsilon}{r}$ if necessary (that is, by taking $\frac{\varepsilon}{r} L$ instead of $L$ ), we can guarantee that $r \leq \varepsilon$. Finally, taking the unit subsegment of the line $L(t)$ with midpoint $P(t)$ for each $t$ we get a continuous motion of a unit line segment in a compact subset of zero Lebesgue measure inside a ball of radius $\frac{1}{2}+\varepsilon$.

Remark 1.6. In the proof of Theorem 1.1 we give an explicit construction for a continuously parametrized Besicovitch set with zero measure. There is a shorter way to prove the existence of such a set: Take a classical compact Besicovitch set $B$ with zero measure and with unit segments in $\mathbf{R}^{n}$. Let $X$ be the metric space of the unit line segments in $\mathbf{R}^{n}$ equipped with the Hausdorff metric and let $K$ consist of those segments of $X$ that are contained in $B$. Then $K$ is a compact subset of $X$. It is well known that every compact metric space is a continuous image of the Cantor set $C$. Hence we have a continuous map $g: C \rightarrow X$ so that $g(C)=K$. All we need to do is to extend this map continuously to $[0,1]$ so that the union of the segments of $g([0,1])$ still has measure zero. Let $(a, b)$ be a complementary interval of $C$. Moving $g(a)$ smoothly to $g(b)$ in such a way that the diameter of $g([a, b])$ is at most twice the distance between $g(a)$ and $g(b)$ gives the desired extension since the union of the new segments will be a smooth 2-dimensional surface having zero measure. Since there are only countably many complementary intervals of $C$ we have the claim.

\section{Lines parametrized continuously by their direction}

In the previous section we constructed lines in every direction in $\mathbf{R}^{n}(n \geq 3)$ so that they can be parametrized continuously by time and their union is small. In this section we parametrize lines continuously by their direction and show, by using simple algebraic topological arguments, that in this case the union must be huge. We denote by $S^{n-1}$ the unit sphere in $\mathbf{R}^{n}$.

Theorem 2.1. Let $L: S^{n-1} \rightarrow\left\{\right.$ lines of $\left.\mathbf{R}^{n}\right\}(n \geq 2)$ be a continuous map such that for each $d \in S^{n-1}$ the line $L(d)$ is parallel to $d$. Then $\mathbf{R}^{n} \backslash \bigcup_{d \in S^{n-1}} L(d)$ is bounded.

Proof. As in the proof of Corollary 1.5, we take a continuous $P: S^{n-1} \rightarrow \mathbf{R}^{n}$ so that $P(d) \in L(d)$ for every $d \in S^{n-1}$ and $P\left(S^{n-1}\right)$ is contained in a ball $B$. Let $O$ be the center of $B$.

We will verify that $\bigcup_{d \in S^{n-1}} L(d) \supset \mathbf{R}^{n} \backslash B$. For this it is enough to show that for any sphere $S$ centered at $O$ and having at least as big radius as $B$ we have $S \subset \bigcup_{d \in S^{n-1}} L(d)$.

For any $d \in S^{n-1}$ the point $P(d)$ is surrounded by $S$, and therefore the halfline in direction $d$ starting from $P(d)$ intersects $S$ in a unique point $Q(d)$. Let $F(d) \in S^{n-1}$ be the direction of the vector from $O$ to $Q(d)$. Since $Q(d) \in L(d)$, all we have to show is that $F$ is onto.

The continuity of $P$ implies that $F: S^{n-1} \rightarrow S^{n-1}$ is continuous. Since $P(d)$ and $O$ are surrounded by $S$ and $Q(d) \in S$, we cannot have $F(d)=-d$. This gives (see e.g. $[6, \mathrm{XV}, 1.2(1)])$ that $F$ and the identity map on $S^{n-1}$ are homotopic. Note that any continuous self-map of $S^{n-1}$ which is not onto, is null-homotopic (see e.g. [6, XV, $1.2(1)]$ ). On the other hand, by Brouwer's Theorem (see e.g. [6, XVI, Theorem 2.1]), 
the identity map on $S^{n-1}$ is not null-homotopic. Hence $F$ must be surjective, which completes the proof.

If $n$ is odd then we can say even more: the lines cover the whole $\mathbf{R}^{n}$.

Theorem 2.2. Let $L: S^{n-1} \rightarrow\left\{\right.$ lines of $\left.\mathbf{R}^{n}\right\}(n \geq 2)$ be a continuous map such that for each $d \in S^{n-1}$ the line $L(d)$ is parallel to $d$. If $n$ is odd then $\bigcup_{d \in S^{n-1}} L(d)=$ $\mathbf{R}^{n}$.

Proof. Again we take a continuous $P: S^{n-1} \rightarrow \mathbf{R}^{n}$ so that $P(d) \in L(d)$ for every $d \in S^{n-1}$. We will prove that for an arbitrary $q \in \mathbf{R}^{n}$ we find $d \in S^{n-1}$ so that $q \in L(d)$. If $q \in P\left(S^{n-1}\right)$ then there is nothing to prove. Thus we may suppose that $q \notin P\left(S^{n-1}\right)$. We define a continuous map $f: S^{n-1} \rightarrow S^{n-1}$ by setting $f(d)$ as the direction of the vector from $q$ to $P(d)$ for every $d \in S^{n-1}$. By a classical result (which follows easily from the famous theorem of Poincare and Brouwer stating that there is no continuous non-vanishing tangent vector field on any $S^{2 k}$ (see for example [6, XVI, Theorem 3.3 and Corollary 3.4])), every continuous $g: S^{n-1} \rightarrow S^{n-1}$ has a fixed point or sends a point to its antipode provided that $n$ is odd. This implies the existence of $d \in S^{n-1}$ so that either $f(d)=d$ or $f(d)=-d$. Since both imply that $q \in L(d)$, the proof is complete.

The following example shows that for every even $n$ Theorem 2.2 is false and we cannot say more than Theorem 2.1 in this case.

Example 2.3. Let $n$ be a positive even number and for any $d=\left(d_{1}, \ldots, d_{n}\right) \in$ $S^{n-1}$ let $L(d)$ be the line through $\left(d_{\frac{n}{2}+1}, \ldots, d_{n},-d_{1}, \ldots,-d_{\frac{n}{2}}\right)$ in direction $d$. Clearly, $L$ is continuous and $L(d)$ is a tangent line of the open unit ball $B(0,1)$ centered at the origin. Hence we find a continuous map $L: S^{n-1} \rightarrow\left\{\right.$ lines of $\left.\mathbf{R}^{n}\right\}$ so that for each $d \in S^{n-1}$ the line $L(d)$ is parallel to $d$ and $\bigcup_{d \in S^{n-1}} L(d)$ is disjoint from $B(0,1)$. (Applying the argument of Theorem 2.1 gives that $\bigcup_{d \in S^{n-1}} L(d)$ covers the complement of $B(0,1)$, and therefore we have $\bigcup_{d \in S^{n-1}} L(d)=\mathbf{R}^{n} \backslash B(0,1)$.)

In the proofs of Theorems 2.1 and 2.2 we heavily used the fact that $L$ is defined on the whole $S^{n-1}$, which caused topological obstacles to obtaining a small union. One might hope to obtain a small union if $L$ is defined only on an open subset of $S^{n-1}$. The following theorem shows that even in this case the union of lines is large. For that we need a topological lemma, which is surely well known but for completeness we present a proof.

Notation 2.4. For any $a \in S^{n-1}$ and angle $r>0$ let

$$
V(a, r)=\left\{d \in S^{n-1}: \angle(d, a) \leq r\right\} \quad \text { and } \quad W(a, r)=\left\{d \in S^{n-1}: \angle(d, a)=r\right\},
$$

where the angle between $d$ and $a$ is denoted by $\angle(d, a)$.

Lemma 2.5. Let $a \in S^{n-1}$ and $0<\varepsilon<r<\frac{\pi}{2}$. Suppose that $f: V(a, r) \rightarrow S^{n-1}$ is a continuous map for which $\angle(d, f(d))<\varepsilon$ for any $d \in V(a, r)$. Then $f(V(a, r)) \supset$ $V(a, r-\varepsilon)$.

Proof. Suppose that $b \in V(a, r-\varepsilon)$ but $b \notin f(V(a, r))$. Let $X=S^{n-1} \backslash\{b,-b\}$. For $x \in V(a, r+\varepsilon) \backslash V(a, r-\varepsilon)$ let $g(x)$ be the closest point of $W(a, r)$ to $x$. Extend $g$ to a continuous retraction $X \rightarrow W(a, r)$. Then for any $d \in W(a, r)$ we have

$$
\angle(d, g(f(d))) \leq \angle(d, f(d))+\angle(f(d), g(f(d))<\varepsilon+\varepsilon<2 r .
$$


This implies that $\left.g \circ f\right|_{W(a, r)}$ is homotopic to the identity of $W(a, r)$. Since $W(a, r)$ is an $(n-2)$-dimensional sphere we see that $\left.g \circ f\right|_{W(a, r)}$ is not null-homotopic. On the other hand, $g \circ f$ is a continuous extension of $\left.g \circ f\right|_{W(a, r)}$ to $V(a, r)$. The fact that $V(a, r)$ is homeomorphic to an $(n-1)$-dimensional ball implies that $\left.g \circ f\right|_{W(a, r)}$ is null-homotopic, which is a contradiction.

Theorem 2.6. Suppose that $U \subset S^{n-1}$ has non-empty interior and $L: U \rightarrow$ \{lines of $\left.\mathbf{R}^{n}\right\}(n \geq 2)$ is a continuous map such that for each $d \in U$ the line $L(d)$ is parallel to $d$. Then $\bigcup_{d \in U} L(d)$ contains an unbounded open set.

Proof. By taking a compact subset of $U$ with non-empty interior if necessary, we may suppose that $U$ is compact. The proof starts as that of Theorem 2.1. We choose a continuous map $P: U \rightarrow \mathbf{R}^{n}$ so that $P(d) \in L(d)$ for every $d \in U$ and select a ball $B \supset P(U)$ and a sphere $S$ centered at the center $O$ of $B$ having radius at least as large as that of $B$. For any $d \in U$, we define $f(d)$ as the direction of the vector from $O$ to the unique intersection $Q(d)$ of $S$ and the halfline in direction $d$ starting from $P(d)$. Note that $Q(d) \in L(d)$ for any $d \in U$.

Pick $a \in U$ and $0<\varepsilon<r<\frac{\pi}{2}$ so that $V(a, r) \subset U$. It is easy to see that if the radius of $S$ is larger than some $R$, which depends only on $\varepsilon$ and the radius of $B$, then $\angle(d, f(d))<\varepsilon$ for any $d \in U$. By Lemma 2.5, this implies that $f(V(a, r)) \supset$ $V(a, r-\varepsilon)$. So any $x \in \mathbf{R}^{n}$ with $|x-O| \geq R$ and $\angle(x, a) \leq r-\varepsilon$ is covered by a line $L(d)$ for some $d \in U$, which completes the proof.

In all of the above results the direction of a line of $\mathbf{R}^{n}$ is represented as a point of $S^{n-1}$. Since lines with opposite directions are the same, it is also natural to represent the directions of the lines of $\mathbf{R}^{n}$ as the points of the real projective space $\mathbf{R} \mathbf{P}^{n-1}$, which is the same as the set of lines of $\mathbf{R}^{n}$ going through the origin. A map on $\mathbf{R} \mathbf{P}^{n-1}$ can be also considered as an even map on $S^{n-1}$, that is, a map whose values agree on opposite points of $S^{n-1}$. This means that Theorems 2.1, 2.2 and 2.6 also hold on $\mathbf{R} \mathbf{P}^{n-1}$. The only question is whether Example 2.3 is valid for a map defined on $\mathbf{R} \mathbf{P}^{n-1}$. The following theorem shows that the answer is negative: in this case both for odd and even $n$ the lines cover the whole $\mathbf{R}^{n}$.

Theorem 2.7. If $F:\left\{\right.$ lines of $\mathbf{R}^{n}$ through the origin $\} \rightarrow\left\{\right.$ lines of $\mathbf{R}^{n}$, where $n \geq 2$, is a continuous map such that for every line $l$ through the origin the line $F(l)$ is parallel to $l$, then $\bigcup_{l} F(l)=\mathbf{R}^{n}$.

Proof. For any $d \in S^{n-1}$, let $l(d)$ be the line in direction $d$ through the origin and let $L(d)=F(l(d))$. Then $L: S^{n-1} \rightarrow$ \{lines of $\left.\mathbf{R}^{n}\right\}$ is a continuous map such that for each $d \in S^{n-1}$ the line $L(d)$ is parallel to $d$. Moreover, $L$ is even, that is, $L(d)=L(-d)$ for any $d$.

In the same way as in the proof of Theorem 2.2 we define a continuous map $f: S^{n-1} \rightarrow S^{n-1}$ and we need to prove that $f(d)=d$ or $-d$ for some $d$. The fact that $L$ is even implies that $f$ is also even. Thus all we need is the well-known fact in algebraic topology that for any $k$ any even map $f: S^{k} \rightarrow S^{k}$ has a fixed point, which can be proved, for example, as follows: If $f$ is even and does not have a fixed point then $f(d) \neq-d$ for any $d$. This implies (see e.g. [6, XV, $1.2(1)]$ ) that $f$ is homotopic to the identity map on $S^{n-1}$, and therefore its degree is 1 (for definition and basic properties see e.g. $[6,7])$. On the other hand, the degree of an even continuous map 
$S^{k} \rightarrow S^{k}$ is always even (see e.g. [7, Theorem 23.24]). This contradiction completes the proof.

\section{Segments parametrized continuously by their direction}

In this section we show that if, instead of lines, we parametrize unit line segments continuously by their direction then the union of the segments can be small. Our construction is based on a construction of Cunningham [4].

Definition 3.1. By an $\varepsilon$-Cunningham set we mean a simply connected subset $C$ of the plane with Lebesgue measure less than $\varepsilon$ in which a unit line segment can be continuously turned around so that its angle is non-decreasing and it returns to its original position with its ends reversed.

Cunningham [4] proved that $\varepsilon$-Cunningham sets exist for any $\varepsilon>0$. The monotonicity of the angle is not stated in [4] but it follows from the construction in which the segment is always either turned by a positive angle or slid along the line containing the segment.

Notation 3.2. Let $N_{\delta}(H)$ be the open $\delta$-neighborhood of a set $H \subset \mathbf{R}^{2}$, that is,

$$
N_{\delta}(H)=\left\{x \in \mathbf{R}^{2}:|x-y|<\delta \text { for some } y \in H\right\} .
$$

We denote by $s(c, d)$ the unit line segment in $\mathbf{R}^{n}$ with midpoint $c \in \mathbf{R}^{n}$ and direction $d \in \mathbf{R}^{n} \backslash\{0\}$. For $n=2$ an angle $\alpha \in \mathbf{R}$ is sometimes used as the second variable of $s$ instead of $d \in \mathbf{R}^{2} \backslash\{0\}$.

The main result of this section is the following theorem, which provides a Besicovitch set with arbitrarily small measure in $\mathbf{R}^{n}$ parametrized continuously by direction of its unit segments.

Theorem 3.3. For every $n \geq 2$ and $\varepsilon>0$ there exists a continuous map

$$
F: S^{n-1} \rightarrow\left\{\text { unit line segments of } \mathbf{R}^{n}\right\}
$$

so that for any $d \in S^{n-1}$ the segment $F(d)$ is parallel to $d$ and it is the same as $F(-d)$ with reversed ends and $\bigcup_{d \in S^{n-1}} F(d)$ is contained in a compact set $B$ of Lebesgue measure less than $\varepsilon$.

Proof. It is enough to find a continuous even map $f: S^{n-1} \rightarrow \mathbf{R}^{n}$ so that $\bigcup_{d \in S^{n-1}} s(f(d), d)$ has measure less than $\varepsilon$.

Let $C$ be an $\frac{\varepsilon}{2}$-Cunningham set and let $c:[0,1] \rightarrow C$ and $\alpha:[0,1] \rightarrow[0, \pi]$ be continuous functions that parametrize the midpoint and the angle of the segment while it is turned around, respectively. We know that $c(0)=c(1), \alpha(0)=0, \alpha(1)=\pi$ and $\alpha$ is non-decreasing.

Fix $0<\delta<\pi$. In order to prove the $n=2$ case, we replace $\alpha$ by a strictly increasing function. Let $t_{0} \in[0,1]$ be such that $\alpha\left(t_{0}\right)=\pi-\delta$ and let

$$
\beta(t)= \begin{cases}\alpha(t)+\delta t & \text { if } t \leq t_{0} \\ \pi-\delta+\delta t & \text { if } t>t_{0}\end{cases}
$$

It is easy to check that $\beta(0)=0, \beta(1)=\pi, \beta$ is strictly increasing and $\mid \beta(t)-$ $\alpha(t) \mid<\delta$ for any $t \in[0,1]$. This last property implies that $s(c(t), \beta(t))$ is in the $\frac{\delta}{2}$-neighborhood of $s(c(t), \alpha(t))$. Since $\beta:[0,1] \rightarrow[0, \pi]$ is a continuous bijection, its inverse $\beta^{-1}:[0, \pi] \rightarrow[0,1]$ is also a continuous bijection. For any $\phi \in[0, \pi)$ and 
$k \in \mathbf{Z}$, define $g(k \pi+\phi)=c\left(\beta^{-1}(\phi)\right)$. Then $g: \mathbf{R} \rightarrow C \subset \mathbf{R}^{2}$ is $\pi$-periodic and continuous (since $c\left(\beta^{-1}(0)\right)=c(0)=c(1)=c\left(\beta^{-1}(\pi)\right)$, and we have

$$
\bigcup_{\phi \in \mathbf{R}} s(g(\phi), \phi)=\bigcup_{t \in[0,1]} s(c(t), \beta(t)) \subset N_{\frac{\delta}{2}}\left(\bigcup_{t \in[0,1]} s(c(t), \alpha(t))\right) \subset N_{\frac{\delta}{2}}(C) .
$$

Thus, for $n=2$, letting $\varphi(d)$ be the angle between $d$ and a fixed vector and taking $\delta$ small enough, $f(d)=g(\varphi(d))$ gives the desired map.

Now let $n \geq 3$. Since $g: \mathbf{R} \rightarrow C$ is continuous and $\pi$-periodic and $C$ is simply connected, there exists a continuous map $H:[0, \delta] \times \mathbf{R} \rightarrow C$ such that $H(0, \cdot)=c_{0}$ for some $c_{0} \in C, H(\delta, \cdot)=g$ and $H(t, \cdot)$ is $\pi$-periodic for any $t \in[0, \delta]$.

Let $P \subset \mathbf{R}^{n}$ be a fixed 2-dimensional plane through the origin. If we identify $\mathbf{R}^{2}$ with $P$ then $C \subset P \subset \mathbf{R}^{n}$. We denote by $\operatorname{proj}_{P}$ the orthogonal projection onto $P$. For any $d \in S^{n-1}$, let $r(d)=\left|\operatorname{proj}_{P} d\right|$. If $r(d) \neq 0$ denote by $\varphi(d)$ the angle between $\operatorname{proj}_{P} d$ and a fixed non-zero vector of $P$.

The function $g(\varphi(d))$ has most of the required properties but it is not defined when $r(d)=0$. This is why we define $f(d)=g(\varphi(d))$ only when $r(d) \geq \delta$ and extend $f$ to the rest of $S^{n-1}$ using the homotopy $H$. More precisely, we claim that

$$
f(d)= \begin{cases}g(\varphi(d)) & \text { if } r(d) \geq \delta \\ H(r(d), \varphi(d)) & \text { if } 0<r(d)<\delta \quad\left(d \in S^{n-1}\right) \\ c_{0} & \text { if } r(d)=0\end{cases}
$$

has all the required properties. The function $f$ is continuous since $H(\delta, \cdot)=g$ and $H(0, \cdot)=c_{0}$. It is an even function since $\varphi(-d)=\varphi(d)+\pi, r(d)=r(-d)$ and both $g$ and $H(t, \cdot)$ are $\pi$-periodic. It remains to prove that $\bigcup_{d \in S^{n-1}} s(f(d), d)$ has measure less than $\varepsilon$. The fact that $C$ has measure less than $\frac{\varepsilon}{2}$ implies that the set $N_{\frac{\delta}{2}}(C)$ has measure less than $\varepsilon$ if $\delta$ is small enough. Hence it is enough to prove that

$$
\bigcup_{d \in S^{n-1}} s(f(d), d) \subset N_{\frac{\delta}{2}}(C) \times\left[-\frac{1}{2}, \frac{1}{2}\right]^{n-2} .
$$

Moreover, recalling that the midpoints of the unit segments are in $C \subset P$, it is enough to show that

$$
\operatorname{proj}_{P} s(f(d), d) \subset N_{\frac{\delta}{2}}(C) \quad\left(d \in S^{n-1}\right) .
$$

Since $\operatorname{proj}_{P} s(f(d), d)$ is a segment of length $r(d)$ with midpoint $f(d) \in C$, the inclusion (3.3) is clear if $r(d)<\delta$. Otherwise, using (3.1), the inclusion (3.3) follows as

$$
\operatorname{proj}_{P} s(f(d), d) \subset s\left(f(d), \operatorname{proj}_{P} d\right)=s(g(\varphi(d)), \varphi(d)) \subset N_{\frac{\delta}{2}}(C) .
$$

With some extra effort we can get a bit more.

Proposition 3.4. We can also guarantee in Theorem 3.3 that $B$ is simply connected and it is contained in a ball of radius $1+\varepsilon$.

To prove this result, we need some additional properties of Cunningham's construction and also a slight technical improvement.

Notation 3.5. We denote by $B_{n}(r)$ and $\bar{B}_{n}(r)$ the $n$-dimensional open and closed ball centered at the origin with radius $r$, respectively.

Theorem 3.6. (Cunningham [4]) For any $\varepsilon>0$ there exists an $\varepsilon$-Cunningham set $C \subset \bar{B}_{2}(1)$ such that $C$ is a finite union of polygons. 
Here by a polygon we mean the closed body of a non-self-intersecting closed polygonal chain. Note that a finite union of polygons does not have to be a polygon even if the union is simply connected - this may happen if two polygons meet in a single point.

Lemma 3.7. For any $\varepsilon>0$ there exists an open $\varepsilon$-Cunningham set $C \subset B_{2}(1+\varepsilon)$ and $\delta_{0}>0$ such that $N_{\delta}(C)$ is simply connected for any $\delta<\delta_{0}$.

Proof. Let $C_{0} \subset \bar{B}_{2}(1)$ be an $\varepsilon$-Cunningham set such that $C_{0}$ is a finite union of polygons. By adding small polygons around the finitely many points where two polygons meet in a singleton, we can make sure that $C_{0}$ is a polygon. Let $d<\varepsilon$ be less than the smallest distance between disjoint edges of $C_{0}$. Then one can check that $N_{\delta}\left(C_{0}\right)$ is simply connected for any $\delta<\frac{d}{2}$. For small enough $d$ the set $N_{\frac{d}{4}}\left(C_{0}\right)$ has measure less than $\varepsilon$. This implies that we can take $C=N_{\frac{d}{4}}\left(C_{0}\right)$ and $\delta_{0}=\frac{\frac{d}{4}}{4}$.

Proof of Proposition 3.4. By Lemma 3.7 there exists an open $\frac{\varepsilon}{2}$-Cunningham set $C \subset B_{2}\left(1+\frac{\varepsilon}{2}\right)$ such that $N_{\delta}(C)$ is simply connected if $\delta$ is small enough. Starting from this $C$ we use the argument and the notation of the proof of Theorem 3.3. First we claim that by choosing $\delta$ small enough and the homotopy map $H$ properly we can guarantee that

$$
\bigcup_{d \in S^{n-1}} s(f(d), d) \subset B_{n}(1+\varepsilon)
$$

Note that if a unit segment is contained in $C \subset B_{2}\left(1+\frac{\varepsilon}{2}\right)$ then its midpoint must be in $B_{2}(\rho)$, where

$$
\rho^{2}=\left(1+\frac{\varepsilon}{2}\right)^{2}-\left(\frac{1}{2}\right)^{2} .
$$

This implies that both $c$ and $g$ are maps into $C \cap B_{2}(\rho)$. It is well known (see e.g. [5, Ap. to Ch. IX, Problem $4(\mathrm{~h})]$ ) that any connected component of the intersection of two simply connected open subsets of the plane is simply connected. Hence every component of $C \cap B_{2}(\rho)$ is simply connected. Since the range of $c$ is connected this implies that we can choose the homotopy map $H$ so that it is a map into $C \cap B_{2}(\rho)$. Then clearly $f$ is also a map into $C \cap B_{2}(\rho)$. We consider two cases, $r(d)<\delta$ and $r(d) \geq \delta$.

First suppose that $r(d)<\delta$. Using the fact that $\operatorname{proj}_{P} s(f(d), d)$ is a segment of length $r(d)<\delta$ with midpoint $f(d) \in C \cap B_{2}(\rho)$, we get that $\operatorname{proj}_{P} s(f(d), d) \subset$ $B_{2}\left(\rho+\frac{\delta}{2}\right)$. Since the points of $s(f(d), d)$ have distance at most $\frac{1}{2}$ from the plane $P$, this implies that their distance from the origin is at most $\sqrt{\left(\rho+\frac{\delta}{2}\right)^{2}+\left(\frac{1}{2}\right)^{2}}$. By $(3.5)$ this distance is less than $1+\varepsilon$ if $\delta$ is small enough. Thus $s(f(d), d) \subset B_{n}(1+\varepsilon)$ in this case.

Now suppose that $r(d) \geq \delta$. Then $f(d)=g(\varphi(d))$. Assuming that $s(f(d), d)=$ $s\left(f(d), \operatorname{proj}_{P} d\right)$, the inclusion $s(f(d), d) \subset B_{n}(1+\varepsilon)$ is clear. Otherwise, let $Q$ be the 2 -dimensional plane that contains the segments $s\left(f(d), \operatorname{proj}_{P} d\right)$ and $s(f(d), d)$. Then $D=Q \cap B_{n}(1+\varepsilon)$ is a two dimensional disc that contains $s\left(f(d), \operatorname{proj}_{P} d\right)$. Since $Q$ contains a vector orthogonal to $P$ and $f(d) \in P, s\left(f(d), \operatorname{proj}_{P} d\right)$ is on the diagonal of $D$. Hence, in the plane $Q$ the disc centered at $f(d)$ with radius $\frac{1}{2}$ is contained in $D$. Using the fact that $s(f(d), d)$ is in this disc, we have $s(f(d), d) \subset D \subset B_{n}(1+\varepsilon)$, which completes the proof of (3.4). 
Let

$$
B=\left(N_{\frac{\delta}{2}}(C) \times\left[-\frac{1}{2}, \frac{1}{2}\right]^{n-2}\right) \cap B_{n}(1+\varepsilon) .
$$

We claim that (for small enough $\delta$ ) the set $B$ has all the required properties. The projection $\operatorname{proj}_{P}$ is clearly a continuous retraction of $B$ to $N_{\frac{\delta}{2}}(C)$. Since $N_{\frac{\delta}{2}}(C)$ is simply connected, $B$ is also simply connected. Finally, (3.2) and (3.4) imply that $\bigcup_{d \in S^{n-1}} s(f(d), d) \subset B$, which completes the proof.

The following observation shows that $B$ cannot be inside a much smaller ball.

Proposition 3.8. Let $B \subset \mathbf{R}^{n}$ be a Besicovitch set parametrized continuously by the directions of its unit line segments as in Theorem 3.3. Assume that $B \subset$ $B_{n}(1-r)$ for some $0<r<1$. Then $B \supset B_{n}(r)$. Thus in this case the measure of $B$ must be at least the volume of an $n$-dimensional ball of radius $r$.

Proof. We generalize an argument of Cunningham [4]. Suppose that $B \subset \mathbf{R}^{n}$ is the set given by Theorem 3.3, $B \subset B_{n}(1-r)$ and $p \in B_{n}(r) \backslash B$. By extending the segments $F(d)$ to full lines, we get a map like in Theorem 2.7. In particular, we find $d \in S^{n-1}$ so that the line of the segment $F(d)$ contains $p$. Since $p \notin B, F(d) \subset B$ and $F(d)$ is a unit segment, this implies that for one of the endpoints $q$ of $F(d)$ we have $|p-q|>1$. This is a contradiction since $|p| \leq r$ and $|q| \leq 1-r$.

It would be interesting to know how small the set in Theorem 3.3 can be. We do not know the answers to the following questions for any $n \geq 3$.

Questions 3.9. Let $B \subset \mathbf{R}^{n}$ be a Besicovitch set parametrized continuously by the directions of its unit line segments as in Theorem 3.3. Is the interior of $B$ nonempty? Does $B$ have positive Lebesgue measure or at least full Hausdorff dimension? What can we say if the function $F$ is defined only on a subset of $S^{n-1}$ with non-empty relative interior?

\section{References}

[1] Besicovitch, A. S.: Sur deux questions d'integrabilite des fonctions. - J. Soc. Math. Phys. l'Univ. Perm 2, 1920, 105-123.

[2] Besicovitch, A. S.: On Kakeya's problem and a similar one. - Math. Z. 27, 1928, 312-320.

[3] Besicovitch, A. S.: On the fundamental geometrical properties of linearly measurable plane sets of points III. - Math. Ann. 116, 1939, 349-357.

[4] Cunningham, F., JR.: The Kakeya problem for simply connected and for star-shaped sets. Amer. Math. Monthly 78, 1971, 114-129.

[5] Dieudonné, J.: Foundations of modern analysis. - Academic Press, New York-London, 1969.

[6] Dugundu, J.: Topology. - Allyn and Bacon, Inc., Boston, 1966.

[7] Fulton, W.: Algebraic topology: A first course. - Grad. Texts in Math. 153, Springer-Verlag, New York, 1995.

[8] Kakeya, S.: Some problems on maximum and minimum regarding ovals. - Tôhoku Science Reports 6, 1917, 71-88.

[9] Mattila, P.: Geometry of sets and measures in Euclidean spaces: Fractals and rectifiability. - Cambridge Univ. Press, Cambridge, 1995.

[10] O'NeIL, T. C.: A note on the parameterisation of Besicovitch sets. - arXiv:0901.3456v1, 2009.

[11] TAO, T.: A remark on the Kakeya needle problem. - blog post, http://terrytao.wordpress.com/ 2008/12/31/a-remark-on-the-kakeya-needle-problem/

Received 15 December 2009 